\title{
Power Wheelchair Virtual Reality Simulator with Vestibular Feedback
}

\author{
Guillaume Vailland*, Yoren Gaffary, Louise Devigne, Valérie Gouranton, Bruno Arnaldi, Marie Babel
}

University of Rennes, INSA Rennes, Inria, CNRS, Irisa, France

Corresponding Author Email: guillaume.vailland@insa-rennes.fr

https://doi.org/10.18280/mmc_c.811-407

Received: 19 August 2020

Accepted: 11 October 2020

\section{Keywords:}

power wheelchair simulator, multisensory feedback, vestibular feedback, sense of presence, cyber-sickness, user study

\begin{abstract}
Autonomy and the ability to maintain social activities can be challenging for people with disabilities experiencing reduced mobility. In the case of disabilities that impact mobility, power wheelchairs can help such people retain or regain autonomy. Nonetheless, driving a power wheelchair is a complex task that requires a combination of cognitive, visual and visuo-spatial abilities. In practice, people need to pass prior ability tests and driving training before being prescribed a power wheelchair by their therapist. Still, conventional training in occupational therapy can be insufficient for some people with severe cognitive and/or visio-spatial functions. As such, these people are often prevented from obtaining a power wheelchair prescription from their therapist due to safety concerns. In this context, driving simulators might be efficient and promising tools to provide alternative, adaptive, flexible, and safe training. In previous work, we proposed a Virtual Reality (VR) driving simula-integrating vestibular feedback to simulate wheelchair motion sensations. The performance and acceptability of a VR simulator rely on satisfying user Quality of Experience (QoE). Therefore, our simulator is designed to give the user a high Sense of Presence (SoP) and low Cyber-sickness. This paper presents a pilot study assessing the impact of the vestibular feedback provided on user QoE. Participants were asked to perform a driving task whilst in the simulator under two conditions: with and without vestibular feedback. User QoE is assessed through subjective questionnaires measuring user SoP and cyber-sickness. The results show that vestibular feedback activation increases SoP and decreases cyber-sickness. This study constitutes a mandatory step before clinical trials and, as such, only enrolled people without disabilities.
\end{abstract}

\section{INTRODUCTION}

People with disabilities may experience mobility loss that can sometimes limit their ability to move around independently and retain access to social activities. Such limitations can be compensated by mobility aids such as power wheelchairs [10]. However, driving a power wheelchair is a challenging task that relies on good cognitive and visuo-spatial abilities especially when dealing with multiple dynamic obstacles outdoors or with door passing or narrow spaces indoors [22].

Thus, navigating safely requires a significant level of expertise that can be achieved through efficient training, especially for people who have cognitive and visio-spatial disabilities that prevent them from fully managing complex driving situations. Indeed, prescribers need to ensure that the powered mobility aid that they are to prescribe to the user will be operated safely in daily-life. If assessment and/or training is unsuccessful, therapists can decide to prevent wheelchair prescription to a patient for safety reasons.

Toaddress this mobility issue for people who cannot operate a wheelchair safely, there are a plethora of works on driving assistance by means of autonomous solutions such as selfdriving power wheelchairs [20]. However, such solutions are generally not adapted to therapists and end-users expectations and needs. Indeed, such autonomous solutions do not leave a sufficient amount of control to the user, which is however necessary to keep soliciting remaining functions and thus prevent loss of function. In addition, doing a task by oneself instead of having it done by a robot it has been shown empowering [15]. There also exist semi-autonomous solutions such as shared control or guidance systems $[6,9]$ that are more suited to user needs as they give a sufficient amount of control to the user. Indeed, with such systems, the user has full wheelchair control most of the time, and trajectory correction is applied only when there is a danger of collision. However, there are still no such solutions available on the market as they are still at the research stage. Moreover semi-autonomous systems that have currently been developed within research laboratories do not manage all types of complex situations. Indeed, current solutions mainly focus on avoiding obstacles within indoor environments $[6,42]$ and do not yet show sufficiently good performances in more complex situations such as navigation within complex urban environments [34], social interactions with pedestrians [11, 18], and negotiation of ramps or curbs [8]. Furthermore, these systems still require good driving skills by the user [20] and thus require training before they can be used in daily life.

Therefore, an alternative solution to navigation assistance systems is to acquire more driving skills by practising in training sessions, in particular in occupational therapy sessions where the user drives in ecological situations (daily-life situations). Nonetheless, these conventional training methods consisting in using a real wheelchair directly may not be adapted for people with severe visual and/or cognitive impairments [1]. 
Additionally, personalized training methods based on particular scenarios (e.g. at home, in hazardous situations, in crowded places) are too complex and costly to perform. In addition, some people with attention disorders may have great difficulty driving a wheelchair in ecological situations as they often have many distractions, multiple people moving around, and so on.

In this context, driving simulators are emerging to overcome these limitations and widen access to power wheelchair training. Indeed, simulation make it possible to perform various scenarios and assessment conditions within a safe, controllable and reproducible environment.

Virtual Reality (VR) makes it possible to build safe simulations and daily-life based scenarios [4]. Furthermore, VR has been shown to be efficient within a clinical context [5, 14]. Compared to other simulation tools, VR allows user immersion inside the Virtual Environment (VE), providing more Sense of Presence (SoP). SoP is the subjective phenomenon describing the user's sense of "being there" in the VE [35]. In the case of wheelchair driving training, a high SoP will increase user ability to intuitively transpose safe driving skills acquired through virtual scenarios to real life situations [37]. However, completing a navigation task while immersed in a VE induces discomforts [19] often characterized as Cybersickness whose symptoms are similar to motion sickness (e.g. nausea, headaches, dizziness) [29]. Cyber-sickness is a major limitation on VR expansion since it significantly alters simulation comfort and user experience.

In previous work, we presented an innovative power wheelchair driving simulator for wheelchair training in VR [43]. Previous, present and future works dealing with driving simulators are conducted in close collaboration with a rehabilitation center to better match the needs of wheelchair users with therapists, making our simulator relevant for actual end-users such as for the purpose of wheelchair training and assessment of driving abilities. Our simulator includes a mechanical platform that provides vestibular feedback and haptic feedback. The platform is able to simulate accelerations using the "tilt coordination" method [3] resulting from user command (vestibular feedback) or from physical response from the VE such as collisions (haptic feedback).

The objective of this paper is to investigate the contribution of vestibular feedback to SoP and Cyber-sickness in a multisensory driving experience. Indeed, we believe that reproducing real power wheelchair accelerations and centrifugal effects will enhance SoP while reducing perception discordance and Cyber-sickness. This study focuses on the impact of vestibular feedback for able-bodied people. It constitutes a first pilot study that enriches our expectations for future works, a basis for other studies on SoP with vestibular feedback and a necessary step before assessing the same assumptions for people with disabilities. This paper first introduces related work on VR and vestibular feedback. Second, it introduces our experimental study using a VR headset and our vestibular feedback platform. Third, we discuss our results and their validity. Finally, we present the conclusions of our work and future work directions.

\section{Related Work}

This section presents the previous work concerning VR simulators. It then introduces two concepts related to VR experience: SoP and Cyber-sickness, and finally evaluate these concepts through experimental study.

\subsection{VR simulators}

The field of VR regroups all computer-based techniques with the aim of simulating a $3 \mathrm{D}$ VE. Thus, such an environment is controllable, reproducible and safe. VR has major assets for building efficient tools known as simulators, largely used in training [16] and rehabilitation [14] programs. Indeed, VR based simulators make it possible to build user-centered, ecological and specific situations. Therefore, the application of VR to power wheelchair simulation appears to be an excellent way to provide training with customized scenarios [2].

\subsection{Sense of presence}

The efficiency of VR based simulation training strongly depends on SoP. SoP refers to the user's sense of "being there" in the VE [35] and depends on various factors such as spatial presence, involvement and experienced realism [33]. The greater the SoP, the more the user will be involved and will learn from his or her virtual experience [37].

Several research projects have investigated ways to increase SoP. The driving simulator NADS Minisim [41] increases the user awareness component of SoP by providing additional haptic feedback information with the aim of enhancing user understanding of the surrounding environment while driving. Even in the entertainment field, a study of increased presence in cinema with additional feedback reveals positive results on user SoP [27].

Regarding wheelchair simulation, the single screen simulator ViEW [24] addresses SoP with user embodiment methods by representing the user's hand in the VE. The miWe simulator also represents the user's hand and investigates the impact of the use of a new wheelchair controller on SoP [38]. Finally, the addition of a feedback platform in the Virtual Fauteuil simulator [28] seems to contribute to a better immersion, and therefore to a better SoP.

\subsection{Cyber-sickness}

Even though VR is a great tool, early studies revealed that it suffers from Cyber-sickness, a major disadvantage [19]. Indeed, during a VR simulation, the user may experience Cyber-sickness, that is, discomforts similar to motion sickness symptoms (e.g. headaches, dizziness or nausea) [29]. Those discomforts arise from perception conflicts between what the user sees and feels, leading to cognitive mismatches. The greater mismatch between feedback in simulation and what is perceived in real experience, the more severe the Cybersickness.

In the context of a driving simulator, Cyber-sickness is more likely to arise as the user sees himself or herself moving, while not perceiving any physical motion. Niniss and Inoue [26] discuss this phenomenon, but never confirm any theory about the causes. Mahajan et al. [21] also observed Cyber-sickness effects when performing a study involving their wheelchair simulator. However, they did not assess the impact of their simulator platform on participant discomfort.

\subsection{AssessingQoE}

SoP assessment is generally assessed through subjective questionnaires in the literature [25]. Participants' answers to such questionnaires are commonly processed using the Wilcoxon signed rank non-parametric test [44]. Questionnaires 
such as NASA-TLX and IPQ, which aim to evaluate respectively cognitive load during a task and SoP, have been already used by VR and clinical specialists [12]. For instance, the IPQ was administered after users with disabilities used the miWe simulator to measure presence in the VE [38]. They used the IPQ to evaluate presence, spatial presence, involvement and realism separately. The IVEQ questionnaire [39] which tackles VR experience assessment is more recent, but can be used as it has already been validated. This questionnaire covers numerous aspects involved in user experience in VR such as immersion and experience consequences such as Cybersickness.

\section{User Study}

\subsection{Objective and hypotheses}

The objective of this paper is to assess the impact of the vestibular feedback provided by our simulator platform on SoP and Cyber-sickness during a wheelchair driving experience in VR. Positive results of this pilot study would advocate for the relevance of the proposed vestibular feedback modality for increasing user Quality of Experience (QoE). We enrolled only able-bodied participants in this experiment, even though our system is designed for people with disabilities. It is indeed necessary for our Research Ethics Committee that we validate system effectiveness and viability with able-bodied participants through a preliminary study before enrolling people with disabilities and end-users to assess the relevancy of such a simulator in training applications and improving wheelchair driving skills. Indeed, we need to prove and ensure user safety while using the simulator to obtain the Committee's approval and run an experiment with people with disabilities.

Based on previous works and our own VR experience, we defined our hypotheses as follows:

Moreover, testing a hypothesis in a pilot study with ablebodied people before using clinical patients as subjects is a commonly applied procedure $[17,30]$.

H1 The addition of vestibular feedback to visual feedback increases SoP for users. In particular, according to the Igroup project consortium (http://www.igroup.org/pq/ipq/index.php):

H1-Spa This feedback increases spatial presence for users.

H1-Inv This feedback increases user involvement.

H1-Rea This feedback increases experienced realism for users.

H2 The addition of vestibular feedback to the visual feedback decreases sensory conflicts and thus Cyber-sickness.

\subsection{Apparatus}

Our apparatus for this study corresponds to our multisensory power wheelchair driving simulator (see Figures 1 and 2). This simulator uses a Standard R-Net joystick for user input. We chose this device as it is a joystick commonly used to control standard power wheelchairs. Concerning feedback, two devices are used to provide vestibular and visual feedback, respectively. The first device is the vestibular feedback platform, on top of which we set a standard wheelchair seat coming from a standard Salsa M power wheelchair. We provide a vestibular feedback using "tilt coordination". In particular, we render linear accelerations using pitch, and centrifugal effects using roll, depending on both linear and angular speeds.

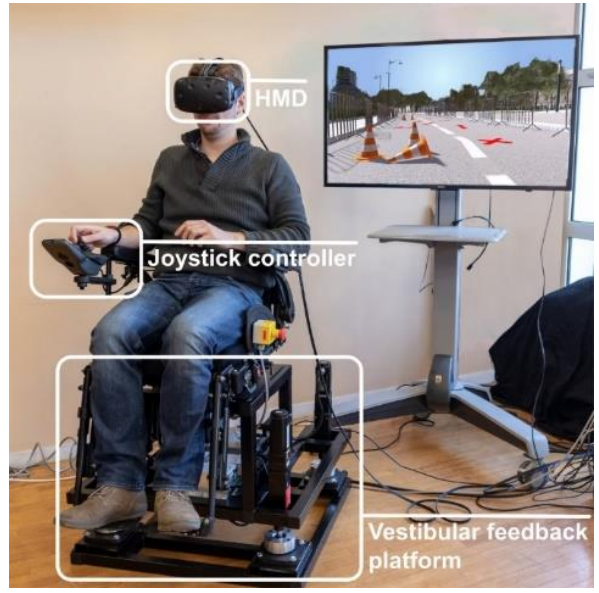

Figure 1. Our power wheelchair driving simulator, composed of an HMD for visual immersion, a joystick to control the virtual power wheelchair, and a vestibular feedback platform

The second device is an HTC Vive HMD. HMD devices make it possible to immerse users in a VE, occulting the real world around them. As it covers all the user vision angles, it also enables users to look backward, which is important for reverse driving. In our case, this would also prevent participants from seeing the vestibular feedback platform movements. Besides, HMDs are largely used in the VR field and provide good head tracking as well as a suitable graphic rendering quality for an immersive experience.

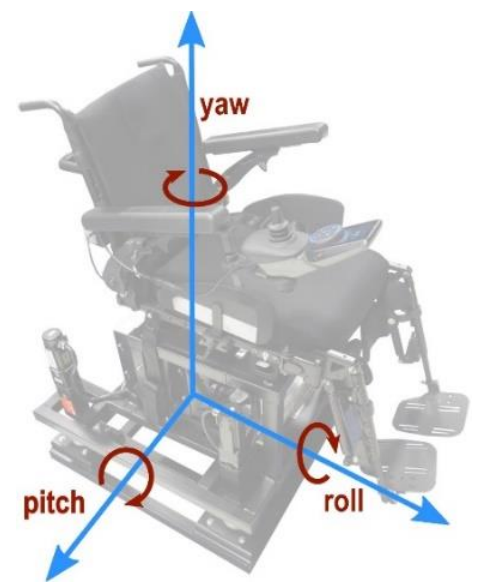

Figure 2. Illustration of feedback platform with rotation axes illustration

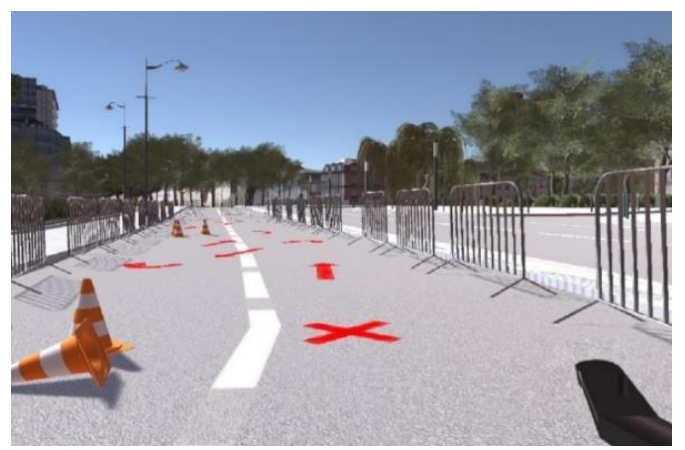

Figure 3. Participant point-of-view during trials. The black part in the bottom-right corner corresponds to the arm of a virtual wheelchair, which is spatially co-located with the real joystick and vestibular feedback platform. 
Concerning the 3D environment, we used a 3D model of a flat square located in a French city. This square was mainly unfamiliar to participants as our experiment took place in anothercity. Weadded assets to this 3D VE to mark out a path that users had to follow in the experiment (see Figure 3). As users might not be familiar with power wheelchair driving, the speed limit of the virtual wheelchair was set to $0.86 \mathrm{~m} . \mathrm{s}^{-1}$ which corresponds to a comfortable speed for navigation.

\subsection{Method}

\subsubsection{Participants}

A total of 29, unpaid and able-bodied French speaking participants were enrolled in this experiment. They were all naive to the purpose of the experiment. All of them signed a consent form explaining the running of the experiment to them. It was also explained to them that they could ask to stop the experiment whenever they wanted. In order for our experiment to be reliable, we applied a strict validation policy. This policy will be explained in details in Section 5. In total, we removed 13 participants. Then, 16 people (average age of 28.6, $\mathrm{SD}=$ 12.33, $\min =19, \max =60$ ) were kept in the analysis. 13 of them were males, and 3 were females. 14 were right-handed.

On average, the participants had little experience with virtual reality and dynamic seats. Most of them had an intermediate to high level of experience with video games. Only one participant had an intermediate level of experience with power wheelchair driving. We conducted this experiment with participants from a different city from the one reproduced in our VE. This was intended to prevent participants from noticing differences between the scene and the real place (i.e. the real place was often crowded while no pedestrians were present in the virtual scene). Only one participant knew the real version of the VE in which participants were asked to perform the driving task.

\subsubsection{Experimental conditions}

We aimed to determine the impact of our platform's vestibular feedback on SoP and Cyber-sickness during a wheelchair driving task in VR. For this experiment, we identified two experimental conditions:

CV No vestibular feedback. Participants were only provided with $\boldsymbol{V}$ isual feedback through the HMD.

CM With vestibular feedback. Participants were provided with visual and vestibular Multisensory feedback.

Each participant completed two trials during this experiment, one for each of the two experimental conditions. The order of presentation was counterbalanced across participants (Eight participants were presented with $\mathbf{C V}$ first, and eight with $\mathbf{C} M$ first).

\subsubsection{Measures}

In order to assess our hypotheses and the impact of our platform vestibular feedback on user QoE, we collected subjective evaluations from participants using three subjective questionnaires, all previously validated in French:

The NASA Task Load Index (NASA-TLX) [13]. This questionnaire was used to determine whether the presence of the vestibular would alter the cognitive load of the user.

The Igroup Presence Questionnaire (IPQ) [32]. This questionnaire allows spatial presence, involvement, and experienced realism to be assessed.

The Immersive Virtual Environments Questionnaire (IVEQ) $[39,40]$. We used all measures concerning immersion, presence, engagement, and experience effects.

The participants completed each of the three questionnaires after each experimental condition.

\subsubsection{Experimental procedure}

Participants were asked to sit on the wheelchair seat attached to the vestibular feedback platform. First, we asked them to attach a seat belt mounted on the platform as a safety measure. We indicated the presence of a big red button, attached to the side of the platform, and that they could press it to immediately shut down the platform if needed.

They received instructions on the use of the joystick controller to operate the virtual power wheelchair in the VE. They also received instructions on the virtual path they had to follow. This path was indicated to the user in the VEby means of red arrows on the floor. It consisted in linear movements, slight curves, large curves, and a stop, a U-turn and finally a reverse driving and parking maneuver. See Section 4.3.5 for additional details (see Figure 4). This set of maneuvers was intended to ensure that participants would perceive various visual and vestibular feedback experiences during the driving task in each trial. The participants were informed that they would have to complete this path twice, and that they would be asked to fill in questionnaires after each time they completed it. Participants did not receive any information about the type of feedback they would have for each trial and about the platform itself. Thereafter, they could ask any questions they had.

Participants were equipped with earmuffs to prevent them from hearing the platform moving or not moving during the trial. They were not able to see the platform either moving or not moving during the trial due to the wearing of the HMD to display the VE. They were given time to familiarize themselves with the VEbefore starting the experiment. They were invited to start the driving task whenever they were ready to. The trial automatically stopped once they reached the end of the path. They were then taken to another room to fulfill the questionnaires assessing user experience in this first trial. This prevented them from seeing the vestibular feedback platform moving when changing the experimental condition. Once they had completed the questionnaires, they performed the second trial. At the end of the second trial, they filled in the same questionnaires again to evaluate their QoE in the second trial.

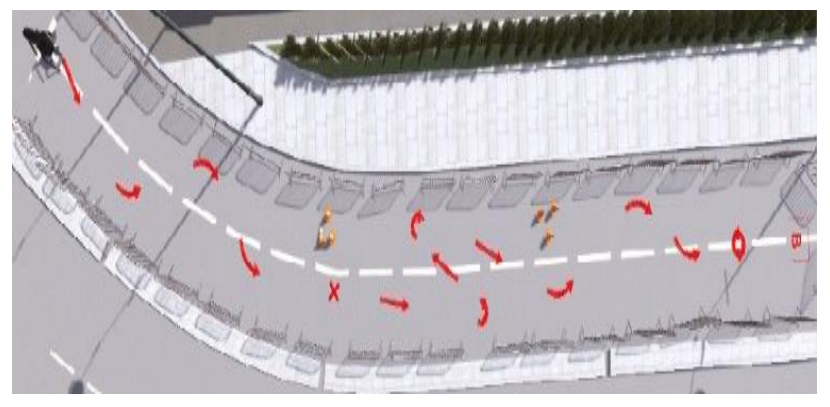

Figure 4. Top view of the path the participants had to complete in both experimental conditions

\section{Results and Analysis}

This section presents our validation policy to ensure that our results were unbiased for the analysis. Then, we will present the results obtained through the three questionnaires for the analysis of SoP and Cyber-sickness, according to the two investigated experimental conditions. 


\subsection{Validation policy}

Analyzing subjective user perceptions such as the SoP required the experiment to be unbiased regarding (1) knowledge related to the vestibular feedback provided and (2) perceptive biases that could have occurred during the experiment. We removed two participants from the experiment after they reported having previously observed the vestibular feedback platform while operating during demonstrations. We then removed additional participants according to three identified perceptive biases they experienced during trials. Firstly, three participants experienced visual discomforts (such as lags or bad quality settings). This might have decreased the immersion. Secondly, six participants might have heard surrounding noises (such as human voices coming from people not involved in the study) despite the earmuff. This might have influenced their SoP along with their focus on the virtual driving task. Finally, two participants decided on their own to park by moving forward against instructions. As such, they did not experienced all expected feedback and their data were discarded from analysis.

After selecting the participants for the analysis, we ensured that the duration of trials completed by the participants was sufficient for them to potentially experience SoP and Cybersickness. Participants completed each trial in $119 \mathrm{~s}$ on average $(\mathrm{SD}=28 \mathrm{~s})$. No significant difference was found between the first and second trials. The duration of trials was sufficient for participants to potentially experience both SoP and Cybersickness as the exposure time does not affect these two components of VR experience [23].

Table 1. Questions presenting significant differences in participant ratings between conditions $\mathrm{C} V$ and $\mathrm{C} M$

\begin{tabular}{|c|c|c|c|c|}
\hline Question & Label & $\mathbf{C} V$ M (SD) & C $M$ M (SD) & p-value \\
\hline IPQ INV3 & "I still paid attention to the real environment. & $-0.75(1.71)$ & $-1.56(1.37)$ & .041 \\
\hline IPQ INV4 & "I was completely captivated by the virtual world. & $0.5(1.66)$ & $1.56(0.70)$ & .026 \\
\hline IPQ REAL2 & $\begin{array}{c}\text { How much did your experience in the virtual environment seem consistent with } \\
\text { your real world experience? }\end{array}$ & $1.19(1.18)$ & $2.12(0.78)$ & .032 \\
\hline IVEQ 1 & "My interactions with the virtual environment seemed natural. " & $6.62(1.65)$ & $7.62(1.41)$ & .049 \\
\hline IVEQ 5 & "The sense of moving around inside the virtual environment was compelling. " & $6.38(2.62)$ & $8.31(1.99)$ & $\ll .01$ \\
\hline IVEQ 15 & $\begin{array}{c}\text { "I became so involved in the virtual environment that it was as if I was inside the } \\
\text { game rather than manipulating a gamepad and watching a screen. }\end{array}$ & $6.31(2.52)$ & $7.88(1.65)$ & .044 \\
\hline IVEQ 61 & "I suffered from vertigo during my interaction with the virtual environment. " & $5.06(2.84)$ & $2.62(2.89)$ & .01 \\
\hline TLX 6 & "How insecure, discouraged, irritated, stressed and annoyed were you?" & $7.06(5.76)$ & $4.06(4.78)$ & .017 \\
\hline
\end{tabular}

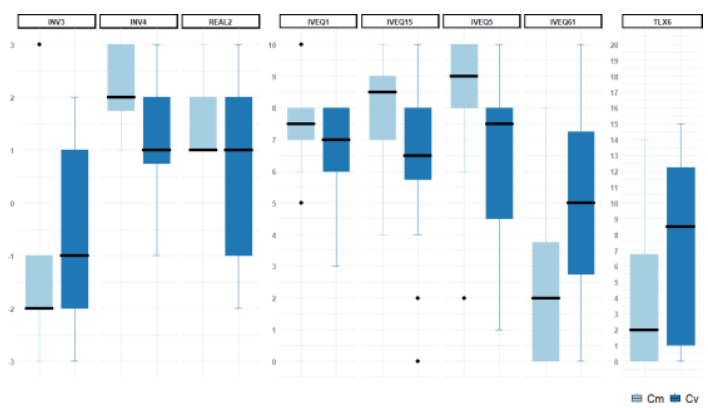

Figure 5. Reported ratings showing significant differences between CV and CM. Scales are displayed in vertical axis.

For each item of the three questionnaires, we compared the intra-subject answers depending on the type of feedback. This section presents only the significant results obtained using a Wilcoxon signed-rank test, related to our hypotheses (see Table 1 and Figure 5). The questionnaire items were presented in their French-validated versions to our participants, but for readability purposes we provide their English-validated equivalent here.

\subsection{Sense of presence}

As we can see in Table 1 and Figure 5, all components of SoP (Spatial presence, Involvement and Experienced realism) presented a significant enhancement through the addition of our vestibular feedback. Thus, these results support our global hypothesis $\mathbf{H 1}$ concerning SoP.

\subsection{Cyber-sickness}

Concerning Cyber-sickness, presented IVEQ, results support H2. Besides, one of our participants reported in open feedback that "the simulation without movements was not pleasant and disrupted the brain".

TLX results suggests that our vestibular feedback globally enhanced quality of the VR experience for our participants. Besides, multiple participants reported in open feedbackhaving preferred the trial with the vestibular feedback.

\section{Discussion}

This section discusses three aspects of our research: the user study, the simulator and observations on SoP and Cybersickness. Future works will also be revealed.

\subsection{User study}

After applying a strict validation policy and despite the number of participants retained for results analysis, we collected and analyzed enough data to obtain significant results. Indeed, the Wilcoxon signed-rank test is a non-parametric test, which implies that no difference between groups could have been established without a consequent effect size. However, a larger set of participants would allow us to investigate the influence of user characteristics (e.g. age, gender, habits, experience) on driving behavior.

Furthermore, this pilot study only involved able-bodied participants, who are not the target population for a wheelchair driving simulator. However, such a study with able-bodied participants constitutes the very first step before assessing our hypotheses with people with disabilities. On the one hand, thisis because we have to validate the relevancy of the simulator modalities provided (here, the vestibular feedback). On the other hand, it is mandatory to perform such a preliminary study with able-bodied participants in order to obtain authorizations from the Research Ethics Committee to initiate clinical 
investigations with people with disabilities. Although the study was not performed with the targeted population, the results remain valid and usable in other projects.

Despite the fact that participants were not acquainted with the city from which the virtual scene had been modelled, the fact that it represents a real world city square contributes to the realism of the environment. Elements used to mark the path for users to follow were inspired by real world objects (barriers and cones), and the arrows were designed to look like tags on the ground. This ensured that the whole environment was as realistic as possible to enhance user SoP. As the path and environment were the same in both conditions, significant differences found for SoP and Cyber-sickness cannot be explained by their variations. The path was also complex enough to ensure the diversity of vestibular feedback presented to users (e.g accelerations, brakes, slight and sharp turns, reverse driving). Furthermore, we chose to limit the experiment driving speed $\left(0.86 \mathrm{~m} . \mathrm{s}^{-1}\right)$ to half the average speed of a real power wheelchair. Indeed, a higher speed induces higher accelerations and thus provides an easier way to stimulate the vestibular system and to reduce Cyber-sickness. Thus, we believe that if vestibular stimulation works at low speed, it will work even better at high speed. Plus, our simulator's target audience is mainly first-time power wheelchair users, who are more likely to drive slower than experienced users. Finally, this scene suffers from a major drawback: the lack of dynamic elements and features. The participants were alone in the scene, while the existing place is usually crowded. The reproduced scene was therefore not entirely ecological. This shows that we ensured that participants had little chance of already knowing the place. It would be interesting to conduct an experiment with pedestrians and cars to better immerse participants in the VE.

\subsection{Simulator and vestibular feedback}

Our simulator benefited from the dual contribution of computer and mechanical scientists to design and build the vestibular feedback platform. We aim to increase our simulator performances over time, in terms of BOTH feedback and physical behavior. It is important to note that we only added vestibular feedback for this study while our feedback platform is also able to simulate haptic and auditory feedback. Indeed, in order to avoid bias and ensure that only the impact of vestibular feedback was evaluated, we turned the haptic feedback off as it is not being evaluated yet. However, once the user is driving at maximum speed, the power wheelchair is no longer subject to accelerations. Thus, the user does not feel any vestibular feedback. This is why we maximized the number of actions that induced acceleration variations during virtual task.

Compared to other types of existing feedback modalities, the main objective of our vestibular feedback is not to give additional information to the user or to help in the navigation [7]. The proposed feedback only provides a consistent and realistic wheelchair behavior in order to decrease Cyber-sickness, increase SoP and thus enhance the VR experience. However, future works on haptic feedback will provide additional relevant information regarding both the navigation and QoE improvements as haptic feedback concerns reactions to the VE.

\subsection{SoP and cybersickness}

Most of the other simulators aim to analyze the issues related to the use of a wheelchair in an urban environment, or to assess training possibilities [24, 28]. To the best of our knowledge, there is no other study investigating the impact of vestibular feedback on SoP and Cyber-sickness in VR.

However, we believe that SoP and Cyber-sickness are key elements in simulator based training. On the one hand, SoP is linked to spatial presence and experienced realism, but also user involvement and motivation, which is known to be an important factor for training [36]. On the other hand, reducing Cybersickness is critical to increase user QoE since most users would not be able to use VR for training purposes due to Cybersickness symptoms. Thus, SoP and Cyber-sickness are key elements to be addressed for a high quality of virtual experience [39].

It is also interesting to point out that while our study concerned a power wheelchair driving task, the presented results remain valid and usable in other projects. In particular, every VR based simulator could benefit from the addition of vestibular feedback. Also, further investigation of vestibular feedback as additional information should be interesting in domains like robot teleoperation and telepresence such as in [31] It could enhance the quality and precision of manoeuvres for real robot experienced operators as they will retrieve realistic sensations.

\section{Conclusion And Future Works}

In this paper, we used our power wheelchair driving simulator to conduct a pilot study assessing the impact of the vestibular feedback provided on user SoP and Cyber-sickness during a driving task in VR.

Participants were asked to drive the virtual power wheelchair along a path in a VE reproducing a real city square. Every participant completed the path twice in a counterbalanced order: one with visual feedback only, and one with visual and vestibular feedback. We used subjective questionnaires (NASA-TLX, IPQ and IVEQ) to assess the quality of the VR experience after completion of each task.

The results validate our stated hypotheses: the addition of the vestibular feedback to the visual feedback provided by the visual display interface rendering the VE increases SoP and decreases Cyber-sickness for users. Therefore, the vestibular feedback contributes to better user QoE while using the simulator to drive a wheelchair in a VE. Those positive results imply that the simulator is more likely to be accepted and used by the targeted population (therapists and end-users). Indeed, the positive impact of the proposed vestibular feedback on increased SoP (by 2.44 10) and reduced Cyber-sickness provides solutions to deal with issues such as user lack of involvement and Cyber-sickness effects that represent important barriers to the use of VR technology by this target population. In a nutshell, our results highlight the importance of providing vestibular feedback in increasing the quality of VR experience and therefore the benefit of our simulator for training and rehabilitation programs.

This first pilot study paves the way towards numerous studies and research. In future works, the impact many parameters such as average speed, virtual environment or participant history should be investigated. In particular, it would be interesting to collect and analyze objective measures (e.g. gaze direction, electrodermal activity) to objectively quantify the impact of the vestibular feedback on SoP and Cyber-sickness and to complete the subjective assessment that we presented in this paper. Concerning participant history and susceptibility to cybersickness we plan to use Motion Sickness Susceptibility 
Questionnaire (MSSQ) with a reduced number of questions by using more precise scales such as Simulator Sickness Questionnaire (SSQ) instead of IVEQ. Also, some guidelines for future works can be drawn from non-significant results with low p-value like the IVEQ-13 item "the simulation without movements was not pleasant and disrupted the brain" which draws our attention to the possible importance of the ecological aspect of the virtual environment.

We also plan to validate our hypotheses for the haptic feedback component of the vestibular feedback platform, corresponding to the vibrations caused by the road on the wheels while driving, among others. Finally, we plan to enroll participants with disabilities to confirm the presented result for target population and make comparisons between their driving behavior and performances with our simulator and a real power wheelchair. This would pave the way for the use of our simulator for training and rehabilitation applications.

\section{ACKNOWLEDGEMENTS}

This work is carried out as part of the INTERREG VA FMA ADAPT project funded by the European Regional Development Fund (ERDF), and is partially funded by the Crowdbot Project, a European Union Horizon 2020 Project.

\section{REFERENCES}

[1] Anstey, K.J., Wood, J., Lord, S., Walker, J.G. (2005). Cognitive, sensory and physical factors enabling driving safety in older adults. Clinical Psychology Review, 25(1): 45-65. https://doi.org/10.1016/j.cpr.2004.07.008

[2] Arlati, S., Colombo, V., Ferrigno, G., Sacchetti, R., Sacco, M. (2019). Virtual reality-based wheelchair simulators: A scoping review. Assistive Technology, pp. 1-12. https://doi.org/10.1080/10400435.2018.1553079

[3] Colombet, F., Dagdelen, M., Reymond, G., Pere, C., Merienne, F., Kemeny, A. (2008). Motion cueing: What is the impact on the driver's behavior. In Proceedings of the Driving Simulation Conference, pp. 171-181.

[4] Cromby, J.J., Standen, P.J., Brown, D.J. (1996). The potentials of virtual environments in the education and training of people with learning disabilities. Journal of Intellectual Disability Research, 40(6): 489-501. https://www.academia.edu/download/30685875/learnin g-disabilities2.pdf

[5] Dascal, J., Reid, M., Ishak, W.W., Spiegel, B., Recacho, J., Rosen, B., Danovitch, I. (2017). Virtual reality and medical inpatients: A systematic review of randomized, controlled trials. Innovations in Clinical Neuroscience, 14(1-2):

14-21 https://www.ncbi.nlm.nih.gov/pmc/articles/PMC537379 1/

[6] Devigne, L., Narayanan, V.K., Pasteau, F., Babel, M (2016). Low complex sensor-based shared control for power wheelchair navigation. In 2016 IEEE/RSJ International Conference on Intelligent Robots and Systems (IROS), pp. 5434-5439. https://doi.org/10.1109/IROS.2016.7759799

[7] Devigne, L., Pasteau, F., Babel, M., Narayanan, V.K., Guegan, S., Gallien, P. (2018). Design of a haptic guidance solution for assisted power wheelchair navigation. In 2018 IEEE International Conference on
Systems, Man, and Cybernetics (SMC), pp. 3231-3236. https://doi.org/10.1109/SMC.2018.00547

[8] Devigne, L., Pasteau, F., Carlson, T., Babel, M. (2019). A shared control solution for safe assisted power wheelchair navigation in an environment consisting of negative obstacles: a proof of concept. In 2019 IEEE International Conference on Systems, Man and Cybernetics (SMC), pp. 1043-1048. https://doi.org/10.1109/SMC.2019.8914211

[9] Erdogan, A., Argall, B.D. (2017). Prediction of user preference over shared-control paradigms for a robotic wheelchair. In 2017 International Conference on Rehabilitation Robotics (ICORR), pp. 1106-1111. https://doi.org/10.1109/ICORR.2017.8009397

[10] Fomiatti, R., Richmond, J., Moir, L., Millsteed, J. (2013). A systematic review of the impact of powered mobility devices on older adults' activity engagement. Physical \& Occupational Therapy in Geriatrics, 31(4): 297-309. https://doi.org/10.3109/02703181.2013.846451

[11] Hansen, S.T., Svenstrup, M., Andersen, H.J., Bak, T. (2009). Adaptive human aware navigation based on motion pattern analysis. In RO-MAN 2009-The 18th IEEE International Symposium on Robot and Human Interactive Communication, pp. 927-932. https://doi.org/10.1109/ROMAN.2009.5326212

[12] Hart, S.G. (2006). Nasa-Task Load Index (NASA-TLX); 20 Years Later. Proceedings of the Human Factors and Ergonomics Society Annual Meeting, 50(9): 904-908. https://doi.org/10.1177\%2F154193120605000909

[13] Hart, S.G., Staveland, L.E. (1988). Development of NASA-TLX (Task Load Index): Results of empirical and theoretical research. In Advances in Psychology, 52: 139-183. https://doi.org/10.1016/S0166-4115(08)623869

[14] Howard, M.C. (2017). A meta-analysis and systematic literature review of virtual reality rehabilitation programs. Computers in Human BEHAVIOR, 70: 317-327. https://doi.org/10.1016/j.chb.2017.01.013

[15] Hurst, A., Tobias, J. (2011). Empowering individuals with do-it-yourself assistive technology. In The Proceedings of the 13th International ACM SIGACCESS Conference on Computers and Accessibility, pp. 11-18. https://doi.org/10.1145/2049536.2049541

[16] Inman, D.P., Loge, K., Leavens, J. (1997). VR Education and rehabilitation. Communication of the ACM, 40(8): 53-58. https://doi.org/10.1145/257874.257886

[17] John, N.W., Pop, S.R., Day, T.W., Ritsos, P.D., Headleand, C.J. (2017). The implementation and validation of a virtual environment for training powered wheelchair manoeuvres. IEEE Transactions on Visualization and Computer graphics, 24(5): 1867-1878. https://doi.org/10.1109/TVCG.2017.2700273

[18] Kruse, T., Pandey, A. K., Alami, R., Kirsch, A. (2013). Human-aware robot navigation: A survey. Robotics and Autonomous Systems, 61(12): 1726-1743. https://doi.org/10.1016/j.robot.2013.05.007

[19] LaViola Jr, J.J. (2000). A discussion of cybersickness in virtual environments. ACM Sigchi Bulletin, 32(1): 47-56. https://doi.org/10.1145/333329.333344

[20] Leaman, J., La, H.M. (2017). A comprehensive review of smart wheelchairs: past, present, and future. IEEE Transactions on Human-Machine Systems, 47(4): 486499. https://doi.org/10.1109/THMS.2017.2706727

[21] Mahajan, H.P., Dicianno, B.E., Cooper, R.A., Ding, D. 
(2013). Assessment of wheelchair driving performance in a virtual reality-based simulator. The Journal of Spinal Cord Medicine, 36(4): 322-332. https://doi.org/10.1179/2045772313Y.0000000130

[22] Massengale, S., Folden, D., McConnell, P., Stratton, L., Whitehead, V. (2005). Effect of visual perception, visual function, cognition, and personality on power wheelchair use in adults. Assistive Technology, 17(2): 108-121. https://doi.org/10.1080/10400435.2005.10132101

[23] Melo, M., Vasconcelos-Raposo, J., Bessa, M. (2018). Presence and cybersickness in immersive content: Effects of content type, exposure time and gender. Computers \& Graphics, 71: 159-165. https://doi.org/10.1016/j.cag.2017.11.007

[24] Morère, Y., Bourhis, G., Cosnuau, K., Guilmois, G., Rumilly, E., Blangy, E. (2018). ViEW: A wheelchair simulator for driving analysis. Assistive technology, 32(3): 125-135. https://doi.org/10.1080/10400435.2018.1503204

[25] Nichols, S., Haldane, C., Wilson, J.R. (2000) Measurement of presence and its consequences in virtual environments. International Journal of Human-Computer Studies, 52(3): 471-491. https://doi.org/10.1006/ijhc.1999.0343

[26] Niniss, H., Inoue, T. (2006). Electric wheelchair simulator for rehabilitation of persons with motor disability. In Symposium on Virtual Reality VIII (Proceedings), Belém (PA).

[27] Oh, E., Lee, M., Lee, S. (2011). How 4D effects cause different types of presence experience?. In Proceedings of the 10th International Conference on Virtual Reality Continuum and Its Applications in Industry, pp. 375-378. https://doi.org/10.1145/2087756.2087819

[28] Pouvrasseau, F., Monacelli, É., Charles, S., Schmid, A., Goncalves, F., Leyrat, P.A., Malafosse, B. (2017). Discussion about functionalities of the Virtual Fauteuil simulator for wheelchair training environment. In 2017 International Conference on Virtual Rehabilitation (ICVR), $\mathrm{pp}$

$1-7$. https://doi.org/10.1109/ICVR.2017.8007509

[29] Rebenitsch, L., Owen, C. (2016). Review on cybersickness in applications and visual displays. Virtual Reality, 20(2): 101-125 https://link.springer.com/content/pdf/10.1007/s10055016-0285-9.pdf

[30] Recio, A. C., Becker, D., Morgan, M., Saunders, N. R., Schramm, L. P., \& McDonald III, J. W. (2013). Use of a virtual reality physical ride-on sailing simulator as a rehabilitation tool for recreational sports and community reintegration: a pilot study. American journal of physical medicine \& rehabilitation, 92(12): 1104-1109.

[31] Robuffo Giordano, P., Deusch, H., Lächele, J., Bülthoff, H.H. (2010). Visual-vestibular feedback for enhanced situational awareness in teleoperation of UAVs. In 66th American Helicopter Society International Annual Forum $2010 \quad$ pp. 2809-2818 http://hdl.handle.net/11858/00-001M-0000-0013-C0565

[32] Schubert, T.W. (2003). The sense of presence in virtual environments: A three-component scale measuring spatial presence, involvement, and realness. Z. für Medienpsychologie, 15(2): 69-71.

[33] Schuemie, M.J., Van Der Straaten, P., Krijn, M., Van Der Mast, C.A. (2001). Research on presence in virtual reality: A survey. CyberPsychology \& Behavior, 4(2): 183-201. https://doi.org/10.1089/109493101300117884

[34] Simpson, R.C. (2005). Smart wheelchairs: A literature review. Journal of Rehabilitation Research \& Development, 42(4): 423-438.

[35] Slater, M., Wilbur, S. (1997). A framework for immersive virtual environments (FIVE): Speculations on the role of presence in virtual environments. Presence: Teleoperators \& Virtual Environments, 6(6): 603-616. https://doi.org/10.1162/pres.1997.6.6.603

[36] Steinmayr, R., Spinath, B. (2009). The importance of motivation as a predictor of school achievement. Learning and individual differences, 19(1): 80-90. https://doi.org/10.1016/j.lindif.2008.05.004

[37] Stevens, J.A., Kincaid, J.P. (2015). The relationship between presence and performance in virtual simulation training. Open Journal of Modelling and Simulation, 3(02): 41. https://doi.org/10.4236/ojmsi.2015.32005

[38] Tao, G., Archambault, P.S. (2016). Powered wheelchair simulator development: implementing combined navigation-reaching tasks with a $3 \mathrm{D}$ hand motion controller. Journal of Neuroengineering and Rehabilitation, 13(1): 1-13. https://doi.org/10.1186/s12984-016-0112-2

[39] Tcha-Tokey, K. (2018). Conception et évaluation de l'expérience utilisateur en environnement virtuel immersif. Ph.D. Dissertation.

[40] Tcha-Tokey, K., Christmann, O., Loup-Escande, E., Richir, S. (2016). Proposition and validation of a questionnaire to measure the user experience in immersive virtual environments. International Journal of Virtual Reality, 16(1): 33-48

[41] Telpaz, A., Rhindress, B., Zelman, I., Tsimhoni, O. (2015). Haptic seat for automated driving: Preparing the driver to take control effectively. In Proceedings of the 7th International Conference on Automotive User Interfaces and Interactive Vehicular Applications, pp. 23-30. https://doi.org/10.1145/2799250.2799267

[42] Trautman, P., Ma, J., Murray, R.M., Krause, A. (2015). Robot navigation in dense human crowds: Statistical models and experimental studies of human-robot cooperation. The International Journal of Robotics Research, 34(3): 335-356. https://doi.org/10.1177\%2F0278364914557874

[43] Vailland, G., Grzeskowiak, F., Devigne, L., Gaffary, Y., Fraudet, B., Leblong, E., Babel, M. (2019). Usercentered design of a multisensory power wheelchair simulator: towards training and rehabilitation applications. In 2019 IEEE 16th International Conference on Rehabilitation Robotics (ICORR), pp. 7782. https://doi.org/10.1109/ICORR.2019.8779496

[44] Woolson, R.F. (2007). Wilcoxon signed-rank test. Wiley encyclopedia of clinical trials, 1-3. https://doi.org/10.1002/9780471462422.eoct979 